Saudi Journal of Medical and Pharmaceutical Sciences

Abbreviated Key Title: Saudi J Med Pharm Sci

ISSN 2413-4929 (Print) |ISSN 2413-4910 (Online)

Scholars Middle East Publishers, Dubai, United Arab Emirates

Journal homepage: http://scholarsmepub.com/sjmps/

Original Research Article

\title{
Forskolin Ameliorates Scopolamine Induced Memory Impairment in Rats
}

\author{
Angad Patole, Dipak Lamdhade, Sunil Dewani, Bhushan Gandhare*, Jyotiranjan Raul \\ Department of Pharmacology, Institute of Pharmaceutical Education and Research, Boragon (Meghe), Wardha, Maharashtra, India
}

\begin{tabular}{ll}
\hline DOI: $10.36348 /$ sjmps.2019.v05i12.008 & | Received: 06.12.2019| Accepted: 13.12.2019| Published: 18.12 .2019 \\
*Corresponding author: Bhushan Gandhare &
\end{tabular}

Abstract

Objective: The present investigation was aimed to assess the effect of Forskolin (FL) against cognitive impairment produced by scopolamine in rats using various behavioral models such as Y-maze, Novel object recognition test (NORT) and some biochemical markers of Alzheimer's disease. Methods: Rats were assigned to six different groups, each group consisting of six animals. The normal animals received distilled water, $10 \mathrm{ml} / \mathrm{kg}$ per orally (p. o.), FL (250, 500,750 $\mu \mathrm{g} / \mathrm{kg}$, p. o.) was administered once daily for two weeks. One the last day of treatment, $90 \mathrm{~min}$. post- administration of the last dose of forskolin, amnesia was produced in rats by administration of scopolamine $(3 \mathrm{mg} / \mathrm{kg}$ ) intraperitonealy (i.p.). Then rats were trained to Y-maze and NORT protocol. Short term memory behavioral responses were recorded after 90 min of training session (retention memory) and $24 \mathrm{~h}$ after training (long term memory). Donepazil (3 mg/kg, p. o.) was used as a standard and was administered for 14 days to positive control groups. Biochemical parameters such as reduced glutathione (GSH), Lipid Peroxidation (MDA) and acetylcholinesterase activity were analyzed. Results: Administration of different doses of FL $(250,500,750 \mu \mathrm{g} / \mathrm{kg})$ once daily for two weeks significantly improved the learning ability and the retention of learned memory in Y-Maze and NORT. Moreover, pre-treatment with FL significantly restored increased lipid peroxidation; normalized glutathione and increased acetylcholinesterase activity. Conclusion: Forskolin enhances cognitive performances of rats against memory impairment by forskolin. Antioxidant activity, mainly inhibition of reactive oxygen species generation by natural diterpenoid compound forskolin advocates its therapeutic efficacy in treating neurodegenerative diseases.

Keywords: Dementia, Forskolin, Scopolamine, Alzheimer's disease.

Copyright @ 2019: This is an open-access article distributed under the terms of the Creative Commons Attribution license which permits unrestricted use, distribution, and reproduction in any medium for non-commercial use (NonCommercial, or CC-BY-NC) provided the original author and source are credited.

\section{INTRODUCTION}

One of the devastating forms of mental illness associated with several factors, marked by intricate, and by assorted mental illness is Alzheimer's disease (AD). The condition is broadly visualized in elderly, and characterized by memory loss and many impairedcognitive functions [1]. "Dementia" multidimensionally encompassesa variety of diseases and conditions that develop when nerve cells collapse structurally and functionally [2]. AD produces an impairment of cognitive abilities that is steep in onset but irreversible in progression. Clinically, $\mathrm{AD}$ is initialized by short term memory loss, contrary to the distant memories which are preserved relatively well. Progressively degeration is marked by impairment of cognitive abilities, namely; ability to calculate, exercise visuospatial skills, and, ideomotor apraxia [3].

Many scientific studies suggest that diterpenes and diterpenes rich food have enhancing effect on memory and cognition in animals as well as humans \& shows neuroprotective effects against AD [4].

Diterpene reverses the $\beta$-amyloid $(\mathrm{A} \beta)$ induced reduction of acetylcholine release from hippocampal brain slices, suggesting potential improvements in learning and memory deteriorated by $\mathrm{A} \beta$. Diterpenes have been known to possess anti-fungal, anti-inflammatory [5], anti-cancer [6], antiviral [7] and antioxidant effects [8].

FL is crystalline solid having off white color, a natural product of diterpenes class. Forskolin (also known by another name coleonol) is obtained from Indian Coleus plant (Coleus forskohlii). Powdered root weighing $0.1 \mathrm{~kg}$ of Indian Coleus plant gives $500 \mathrm{mg}$ of FL residue [9]. This plant and related species have been used traditionally in Brazil, Africa, India and other parts of Asia [10-12]. FL is reported to possess antioxidant property [13]. 
Though various treatment strategies are available the severity and prevalence of these diseases are not yet under control. Currently used treatments, like Acetylcholineesterase inhibitors (AchEIs) \& NMethyl D-Aspartic Acid (NMDA) antagonist, do not cause reduction in the progression of the disease which provides little therapeutic benefits. Accordingly, there is pressing need to find novel \& effective medication or combination of drugs for $\mathrm{AD}$ which is more effective $\&$ safer \& go beyond AchEIs \& NMDA antagonist [14]. Hence, there is growing tendency to use alternative and complementary medicines like herbal extracts consisting of phytochemicals in the management of AD. Therefore present study, evaluated the effect of Forskolin against scopolamine-induced cognitive impairment.

\section{MATERIALS AND METHOD \\ Procurement of Experimental Animals}

Male albino rats (Wistar strain) of age 8-12 months, weighing 150-200 g, bred in the animal house of Institute of Pharmaceutical Education and Research (I.P.E.R.), Wardha, were procured. The rats were kept in polypropylene cages at a temperature of $24 \pm 2^{\circ} \mathrm{C}$ with a relative humidity of $40-60 \%$ and 12 hrs lightdark cycle. The experiments were performed in a sound attenuated environment between 8:00 am and 12:00 noon. Rats were provided balanced food and water $a d$ libitum through out experimental period. All the animals were familiarized to the experimental conditions for week before start of experimental studies.

\section{Chemicals}

Forskolin was obtained from UDCT, Mumbai. Scopolamine was procured from Sigma Aldrich, U.S.A. 5, 5'-dithiobis (2- nitrobenzoic acid)(DTNB), Trichloroacetic Acid (TCA), Thiobarbituric Acid (TBA), n-Heptane, Isobutanol, Pyridine, Sodium Lauryl Sulphate (SLS) were obtained from Loba Chemicals, Mumbai. All other chemicals used for the experimental purpose were of analytical grade. All drug solutions were freshly prepared.

\section{Experimental Design}

Rats were assigned to six different groups, each group containing six animals. Group I (Normal): Animals received distilled water, $10 \mathrm{ml} / \mathrm{kg}$ (p.o.).

Group II served as negative control, Group III, IV and V received FL $(250,500,750 \mu \mathrm{g} / \mathrm{kg}$, p.o. respectively) for two weeks once a daily [13]. On $14^{\text {th }}$ day after $90 \mathrm{~min}$. of the last dose administration, scopolamine ( $3 \mathrm{mg} / \mathrm{kg}$, i.p.) amnesia was produced in rats except Group I rats [13]. Then rats were trained to Y-maze and NORT. Short and long term memories were recorded after 90 min. and $24 \mathrm{~h}$ after training. Donepezil (3 $\mathrm{mg} / \mathrm{kg}$ ) (Group VI) was used as a standard and was administered per orally for two weeks to positive control group [15].

\section{Y - MAZE TEST}

Two-trial Y-maze test is used to study cognitive behavior in rats. The first trial was conducted following the terminal dose of FL (250, 500,750 $\mu \mathrm{g} / \mathrm{kg}$ ). For this trial rats were placed at the start arm so that, rats were in position to explore the start, other arms and novel arm throughout 15-min session. One day after first trial, rats were initially put in one arm, subsequently the sequence (ABCCAB) and the total number of entries in arm were recorded for each rat upto 8-min period. It was considered as spontaneous alternation if rat entered into total three arms on successive choices (ABC, $\mathrm{CAB}$, or BCA, but not $\mathrm{BAB}$ ). Thorough cleaning of maze arms was done with water after each animal to remove residual odors. Spontaneous alteration (\%) on Y-maze (90 min. \& 24 hrs after training) and Total entry (No.) on Y-maze (90 min. \& $24 \mathrm{hrs}$ after training) were measured to determine the spatial memory \& locomotor activity response during the second trial. For the experiment, rats were kept on a restricted food schedule for 14 days. During this period, drug treatment was administered, once a day, and the test was performed one day after the last administration of dose [16].

\section{Novel Object Recognition Test (NORT)}

NORT is based on rat's spontaneous behavior. This assumes that when rat has access to novelty (object or environment), it can elicit approach behaviors in rat. The NORT has been utilised in various ways, but typically it has of two trials. Initially for first trial (T1) the rat is exposed to one or two identical (sample) objects. Once the sample object exposed to the rat, it is returned to home cage for a retention period. For the second trial (T2), the rat was returned to the environment and presented with a familiar and a novel object. When the subject 'remembers' the previous exposure to the familiar object, it will explore the novel object to a greater degree than that of the familiar one [17].

The performance of a given animal in each trial was assessed using two parameters:

1. Time spent with the new (novel) object.

2. Object Recognition Index (ORI) for each animal was expressed by $\mathrm{T}_{\mathrm{N}} /\left(\mathrm{T}_{\mathrm{N}}+\mathrm{T}_{\mathrm{F}}\right)$ ratio one

$\left[\mathrm{T}_{\mathrm{F}}=\right.$ time spent exploring familiar object; $\mathrm{T}_{\mathrm{N}}=$ time spent exploring the novel object].

The closer this ratio gets to one, more the rats spend time exploring the new object. Objects were cleaned with $10 \%$ ethanol solution between trials. Exploration was defined when rat directed the nose to the object at a distance of no more than $2 \mathrm{~cm}$ and/or 
touching the object with the nose or forepaws. Sitting on any object was not considered as exploratory behavior.

\section{Biochemical Assays}

\section{Determination of Reduced Glutathione}

The GSH levels were determined by using the method used by Moron et al., [18]. In brief, $0.1 \mathrm{ml}$ of plasma was precipitated using 5\% trichloroacetic acid (TCA). The precipitate was removed by centrifugation. To an aliquot of the supernatant, $2 \mathrm{ml}$ of 5-5'Dithiobis, 2-nitrobenzoic acid (DTNB) reagent was mixed to adjust the final volume of $3 \mathrm{ml}$. Absorbance was recorded at $412 \mathrm{~nm}$ against a blank which had TCA instead of the sample. The total quantity of reduced glutathione was expressed as $\mu \mathrm{mol} / \mathrm{ml}$ of plasma.

\section{Determination of Lipid Peroxidation}

The TBARS levels were determined as per the spectrophotometric method reported by Biradar et al., [15] Each test tube was added, $0.5 \mathrm{ml}$ of plasma, $0.5 \mathrm{ml}$ of normal saline, $1 \mathrm{ml}$ of $20 \%$ TCA and $0.25 \mathrm{ml}$ of TBA reagent $(200 \mathrm{mg}$ of thiobarbituric acid in $30 \mathrm{ml}$ distilled water and $30 \mathrm{ml}$ of acetic acid). The test tubes content were allowed to boil at $95^{\circ} \mathrm{C}$ for one hour. Then each of the test tube was added, $3 \mathrm{ml}$ of $\mathrm{n}$-butanol and mixed well. These tubes were centrifuged for $10 \mathrm{~min}$. at $3000 \mathrm{rpm}$. The butanol layer was collected and read in a spectrophotometer against reagent blank at $535 \mathrm{~nm}$. TBARS concentration was expressed in terms of nmol of malondialdehyde per milliliter of plasma.

\section{Determination of Acetylcholinesterase Activity (AChE)}

The Acetylcholinesterase levels were estimated as per the method described by Biradar et al., [15] Briefly, to each test tube, $0.4 \mathrm{ml}$ of supernatant (after plasma treatment), $2.6 \mathrm{ml}$ phosphate buffer $(0.1$ $\mathrm{M}, \mathrm{pH}$ 8) \&100 $\mu \mathrm{l}$ of 5, 5'-dithiobis (2-nitrobenzoic acid). The contents of the cuvette are mixed thoroughly absorbance is measured at $412 \mathrm{~nm}$. When absorbance reaches a stable value, it is recorded as the basal reading. Then $20 \mu \mathrm{l}$ of substrate i.e., acetylthiocholine is added and change in absorbance is recorded for a period of $10 \mathrm{~min}$ at intervals of $2 \mathrm{~min}$. Change in the absorbance per $\mathrm{min}$ is thus determined. Results expressed as $\mathrm{nmol} / \mathrm{min} / \mathrm{g}$ of plasma.

\section{STATISTICAL ANALYSIS}

Data were analyzed using Graph Pad Prism 5 for Windows (version 5.02). Results were expressed as Mean \pm SEM. One-way analysis of variance (ANOVA) and Dunnett's T-test was used to test the significance of the difference between the variables in various groups. The $\mathrm{P}$ values of less than 0.05 were considered to be statistically significant.

\section{RESULTS \\ Y-MAZE}

Spontaneous alteration (\%) on Y-maze in Rats

Effect of forskolin on spontaneous alteration

(\%) on Y-maze in rats is indicated in Figure-1. Scopolamine treatment significantly reduced the spontaneous alteration index $\left({ }^{\#} \mathrm{P}<0.001\right)$ in contrast to normal, $90 \mathrm{~min}$. and $24 \mathrm{hrs}$ after training in Y-maze. Spontaneous alteration was significantly increased by treatment with FL $750 \mu \mathrm{g} / \mathrm{kg}(* * * \mathrm{P}<0.001)$, FL 500 $\mu \mathrm{g} / \mathrm{kg}(* * \mathrm{P}<0.01)$ with respect to scopolamine group. Spontaneous alteration was increased in contrast to control group after 90 min. \& 24 hrs by treatment with FL $250 \mu \mathrm{g} / \mathrm{kg}$ but it was not significant statistically.

\section{Total entry (No.) on Y-maze in Rats}

Effect of forskolin in different doses on total entry on Y-maze in rats is indicated in Figure-2. Scopolamine treatment significantly reduced the total entry index ( $\left.{ }^{\#} \mathrm{P}<0.001\right)$ as compared to normal $90 \mathrm{~min}$. and $24 \mathrm{hrs}$ after training in $\mathrm{Y}$-maze. Total entries were significantly increased by treatment with FL $750 \mu \mathrm{g} / \mathrm{kg}$ $(* * \mathrm{P}<0.001), \mathrm{FL} 500 \mu \mathrm{g} / \mathrm{kg}(* \mathrm{P}<0.01)$ as compared to scopolamine group. There was no significant increase in total entry after $90 \mathrm{~min}$. \& $24 \mathrm{hrs}$ by treatment with FL $250 \mu \mathrm{g} / \mathrm{kg}$ in contrast to scopolamine group.

\section{Novel Object Recognition Test (NORT) Time Spent On Novel Object}

Effect of forskolin on time spent on the novel object in NORT is indicated in Figure-3. Scopolamine treatment decreased the time spent on the novel object which was statistically significant $\left({ }^{\#} \mathrm{P}<0.001\right)$ in comparison with normal $90 \mathrm{~min}$. and $24 \mathrm{hrs}$ after training in NORT. The time spent on the novel object was significantly increased by treatment with FL 750 $\mu \mathrm{g} / \mathrm{kg}(* * \mathrm{P}<0.001), \mathrm{FL} 500 \mu \mathrm{g} / \mathrm{kg}(* \mathrm{P}<0.01)$ in contrast to scopolamine group. The time spent on new object was not significantly increased by treatment with FL $250 \mu \mathrm{g} / \mathrm{kg}$ as compared to control (scopolamine) group.

\section{Object Recognition Index in Young Rats}

Effect of different doses of Forskolin on ORI in NORT is indicated in Figure-4. Scopolamine treatment significantly reduced the ORI $\left({ }^{\#} \mathrm{P}<0.001\right)$ in comparison with normal $90 \mathrm{~min}$. and $24 \mathrm{hrs}$ after training in NORT. The ORI was significantly increased by treatment with FL $750 \mu \mathrm{g} / \mathrm{kg}(* * * \mathrm{P}<0.001)$, FL 500 $\mu \mathrm{g} / \mathrm{kg} \quad(* * \mathrm{P}<0.01)$ in contrast to scopolamine group. The ORI was not significantly increased after $90 \mathrm{~min}$. $\& 24 \mathrm{hrs}$ of treatment with FL $250 \mu \mathrm{g} / \mathrm{kg}$ as compared to control.

\section{Biochemical Estimation \\ Determination of Reduced Glutathione (GSH) in Rats}

Effect of forskolin on the level of reduced glutathione in plasma of rats is indicated in Figure-5. The level of reduced glutathione in plasma was 
significantly decreased by scopolamine as compared to normal. Treatment with FL $750 \mu \mathrm{g} / \mathrm{kg}(* * \mathrm{P}<0.001)$, $500 \mu \mathrm{g} / \mathrm{kg}(* \mathrm{P}<0.01)$ significantly restored the level of reduced glutathione in plasma in contrast to scopolamine group. There was no significant decrease in the level of reduced glutathione in plasma after 90 min. \& $24 \mathrm{hrs}$ by treatment with FL $250 \mu \mathrm{g} / \mathrm{kg}$ in comparison with control (scopolamine) group.

\section{Determination of Lipid Peroxidation}

The effect of forskolin treatment on the level of TBARS in the plasma of rats is indicated in Figure- 6 . The levels of TBARS were significantly decreased by scopolamine treatment as compared to normal. Treatment with FL $750 \mu \mathrm{g} / \mathrm{kg}(* * * \mathrm{P}<0.001), 500 \mu \mathrm{g} / \mathrm{kg}$ $(* * \mathrm{P}<0.01), 250 \mu \mathrm{g} / \mathrm{kg}(* \mathrm{P}<0.05)$ significantly restored the decreased level of TBARS in comparison with scopolamine group. There was no significant decreased the level of TBARS in plasma after $24 \mathrm{hrs}$ by treatment with FL $250 \mu \mathrm{g} / \mathrm{kg}$ in contrast to (control) scopolamine group.

\section{Determination of Acetylcholinesterase}

Effect of forskolin on the level of acetylcholinesterase in plasma of rats is indicated in Figure-7. The level of acetylcholinesterase in plasma was significantly decreased by scopolamine as compared to normal. Treatment with FL $750 \mu \mathrm{g} / \mathrm{kg}$ $(* * \mathrm{P}<0.001), \quad 500 \quad \mu \mathrm{g} / \mathrm{kg} \quad(* \mathrm{P}<0.01) \quad$ significantly restored the level of acetylcholinesterase in comparison with scopolamine group. There was no significant effect of FL $250 \mu \mathrm{g} / \mathrm{kg}$ on the level of acetylcholinesterase after $90 \mathrm{~min}$. \& $24 \mathrm{hrs}$ as compared to (control) scopolamine group.

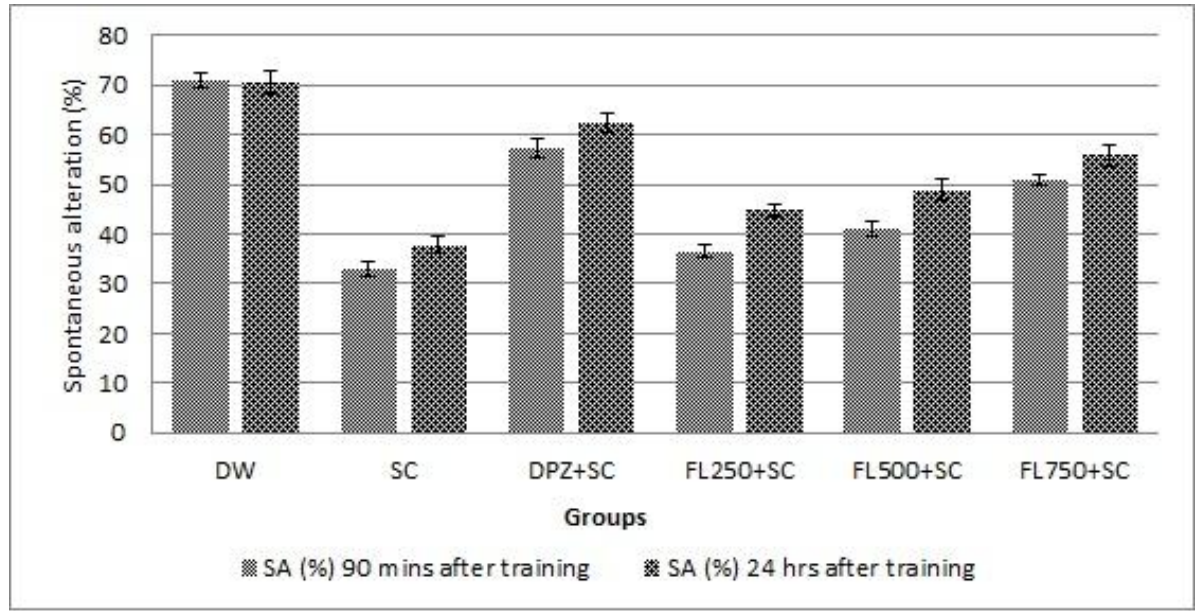

Fig-1: Effect of Forskolin Treatment on Spontaneous alteration (\%) (SA) in Scopolamine groups in Y-maze

Each group consists of 6 animals $(n=6)$. Values are Mean \pm S.E.M. ${ }^{\#} \mathrm{P}<0.001$ as compared to
Normal group. ${ }^{\mathrm{NS}} \mathrm{P}<0.05,{ }^{* *} \mathrm{P}<0.01,{ }^{* * *} \mathrm{P}<0.001$ as compared to Scopolamine group.

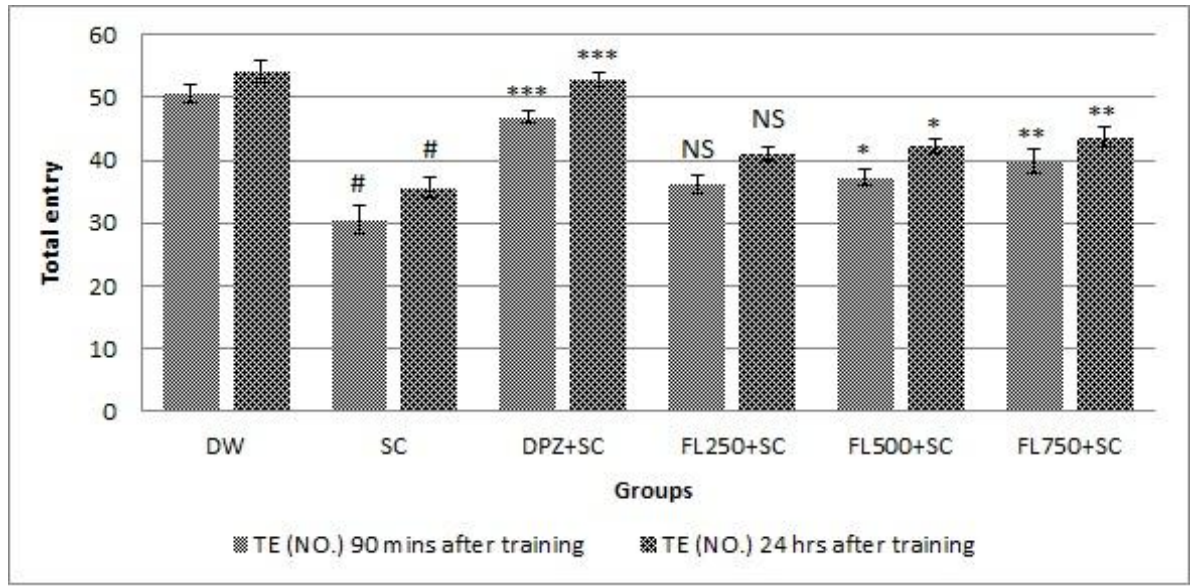

Fig-2: Effect of Forskolin Treatment on Total entry (TE) in Scopolamine groups in Y-maze

Each group consists of 6 animals $(n=6)$. Values are Mean \pm S.E.M. ${ }^{\#} \mathrm{P}<0.001$ as compared to
Normal group. ${ }^{\mathrm{NS}} \mathrm{P}<0.05,{ }^{*} \mathrm{P}<0.01,{ }^{* *} \mathrm{P}<0.001$ as compared to Scopolamine group. 


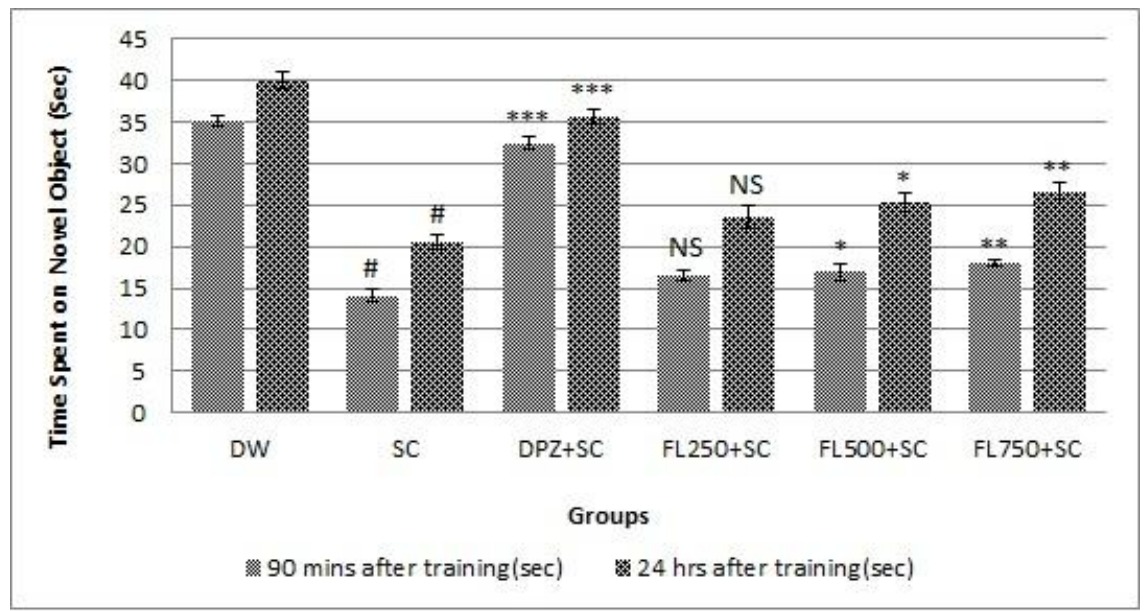

Fig-3: Effect of Forskolin (Fors.) Treatment on Time Spent on Novel Object in Scopolamine Groups

Each group consists of 6 animals $(\mathrm{n}=6)$. Values are Mean \pm S.E.M. ${ }^{*} \mathrm{P}<0.001$ as compared to
Normal group. ${ }^{\mathrm{NS}} \mathrm{P}<0.05,{ }^{*} \mathrm{P}<0.01,{ }^{* *} \mathrm{P}<0.001$ as compared to Scopolamine group.

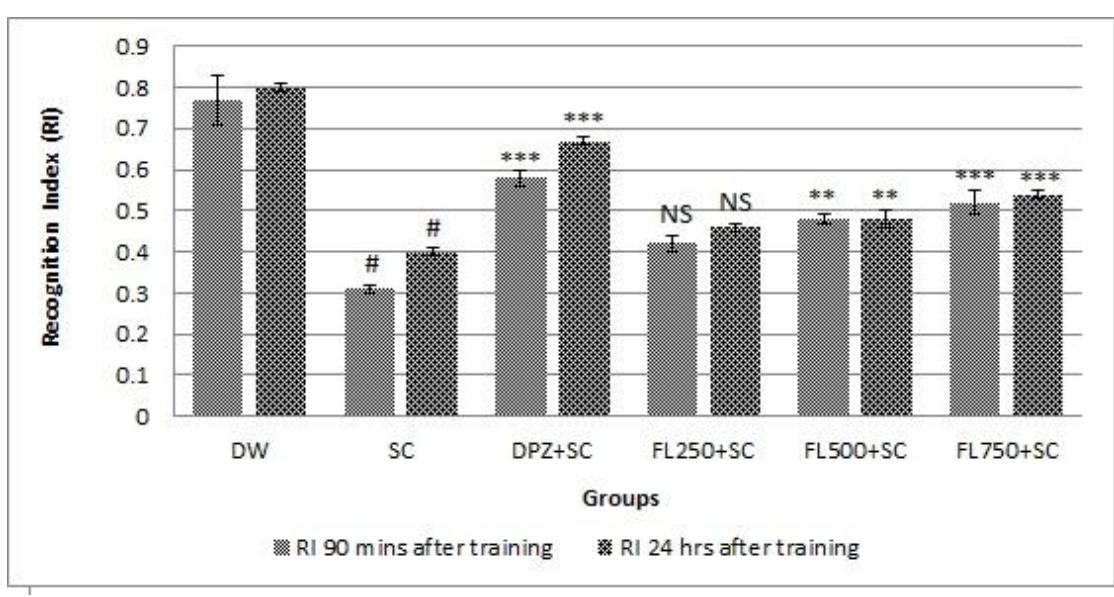

Fig-4: Effect of Forskolin (Fors.) Treatment on Recognition Index (RI) in Scopolamine Groups in NORT

Each group consists of 6 animals ( $\mathrm{n}=6$ ). Values are Mean \pm S.E.M. ${ }^{*} \mathrm{P}<0.001$ as compared to
Normal group. ${ }^{\mathrm{NS}} \mathrm{P}<0.05,{ }^{* *} \mathrm{P}<0.01,{ }^{* * *} \mathrm{P}<0.001$ as compared to Scopolamine group.

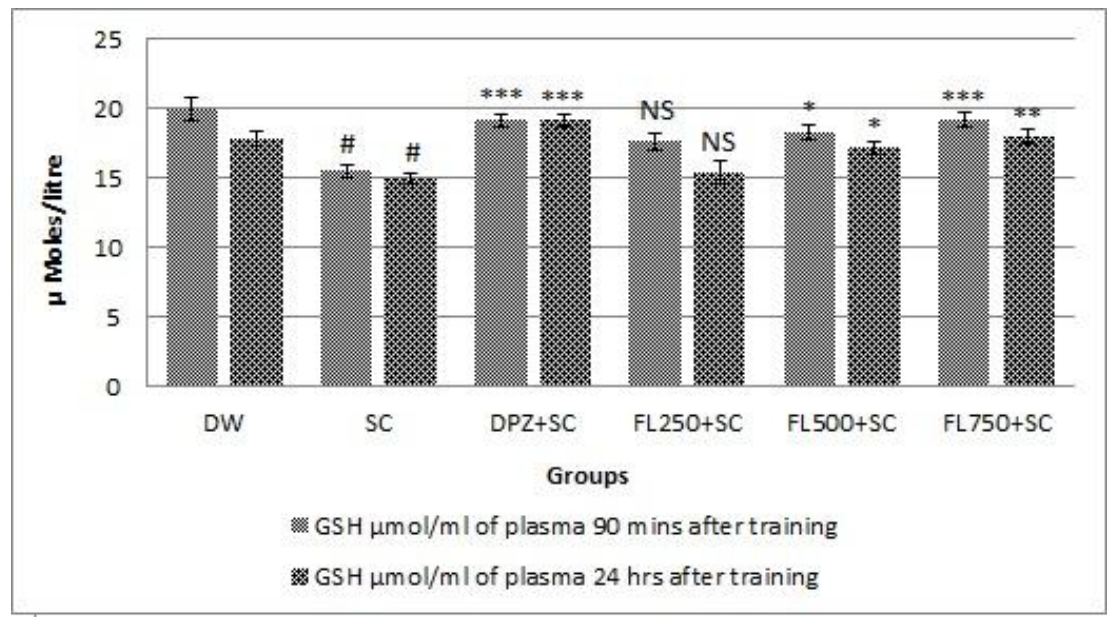

Fig-5: Effect of Forskolin (Fors.) Treatment on the Level of Reduced Glutathione in plasma in Scopolamine Group

Each group consists of 6 animals $(\mathrm{n}=6)$. Values are Mean \pm S.E.M. ${ }^{*} \mathrm{P}<0.001$ as compared to
Normal group. ${ }^{*} \mathrm{P}<0.05,{ }^{* *} \mathrm{P}<0.01,{ }^{* * *} \mathrm{P}<0.001$ as compared to Scopolamine group. 


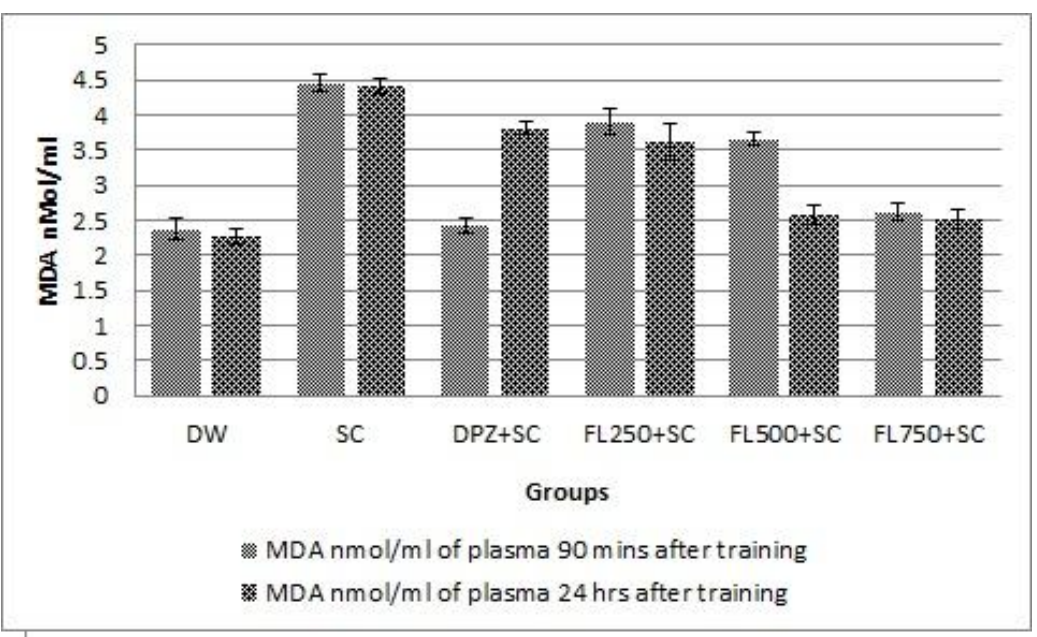

Fig-6: Effect of Forskolin (Fors.) Treatment on the level of TBARS in plasma in Scopolamine Groups

Each group consists of 6 animals $(n=6)$. Values are Mean \pm S.E.M. ${ }^{*} \mathrm{P}<0.001$ as compared to
Normal group. ${ }^{*} \mathrm{P}<0.05,{ }^{* *} \mathrm{P}<0.01,{ }^{* * *} \mathrm{P}<0.001$ as compared to Scopolamine group.

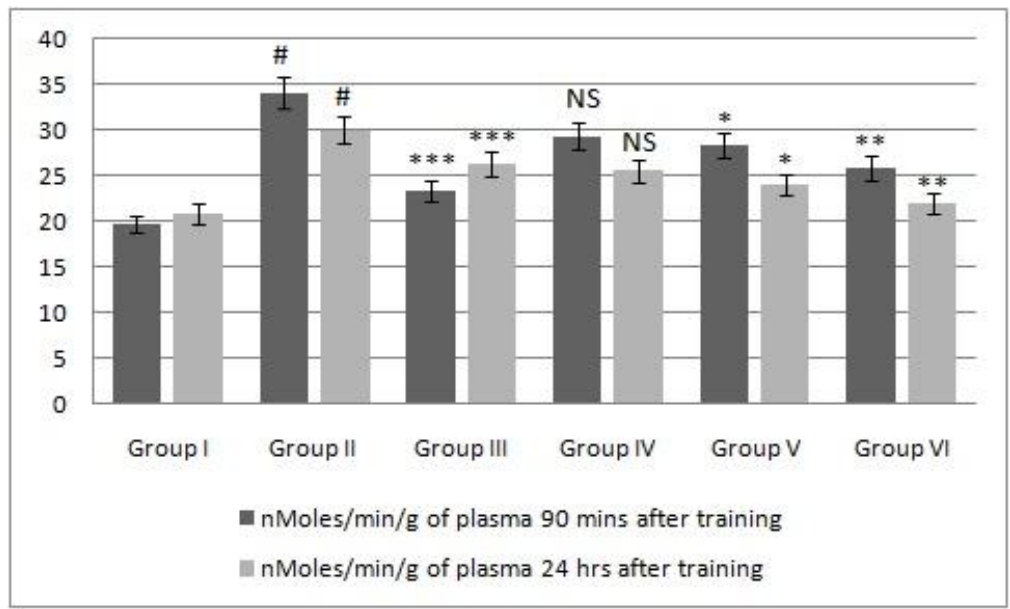

Fig-7: Effect of Forskolin (Fors.)Treatment on the level of acetylcholinesterase in plasma in Scopolamine Groups

Each group consists of 6 animals $(n=6)$. Values are Mean \pm S.E.M. ${ }^{\#} \mathrm{P}<0.001$ as compared to Normal group. ${ }^{\mathrm{NS}} \mathrm{P}<0.05,{ }^{*} \mathrm{P}<0.01,{ }^{* *} \mathrm{P}<0.001$ as compared to Scopolamine group.

\section{DISCUSSION}

Cognitive impairment is a major health issue in the $21^{\text {st }}$ century. AD is the most common root of cognitive impairment and dementia in the elderly, marked by extracellular deposits of $\mathrm{A} \beta$ peptide, neurofibrillary tangles made up of hyperphosphorylated tau, neurodegeneration, and acetylcholine dysfunction. The deposition of soluble $\mathrm{A} \beta$ produces the aggregation. The protein forming amyloid fibrils is reported to be toxic to neurons both in vitro $\&$ in vivo. Since the direct mechanism inducing neurotoxicity by the $A \beta$ peptide remains unclear, the evidence presented in the earlier work suggests that the damage described in AD brain is consistent with some degree of oxidative stress caused by the $\mathrm{A} \beta$ peptide.
Oxygen is necessity of life, but paradoxically, its metabolism produces reactive oxygen species (ROS) as a byproduct which is highly toxic to the cell. Brain tissues from patients which have neurodegenerative disorders, including Parkinson's disease, Alzheimer disease, clearly display raised indices of ROS in the affected brain regions. Brain has high metabolic rate and with no or relatively less capacity of cellular regeneration because of in state of oxidative stress the brain is believed to be susceptible to the destructive effects of ROS. In cases, Alzheimer disease, a variety of indices of ROS damage have been scientifically reported within the definite brain region that undergoes discriminating neurodegeneration [19]. Markers for lipid peroxidation, including malondialdehyde (MDA), and 4hydroxynonenal (4-HNE) as markers for oxidative damage to lipids; isoprostane as a product of the free radical oxidation of arachidonic acid; 8-oxoguanine (8hydroxyguanine) and thymine glycol as indicators of oxidative damage to DNA; and various products of the oxidation of protein and amino acids including hydroxyleucine, hydrovaline, nitrotyrosine and carbonyl 
protein [20]. Normally, the cell has got numerous defenses and repair systems through evolution to deal with increased ROS generation and oxidative damage caused by ROS, but in these situations, the activities of various free radicals scavenging molecules that would normally work against the injurious effect of ROS are reduced. For example catalase, superoxide dismutase (SOD), glutathione peroxidase and reductase (GSHPx \& GSHRd), all these antioxidant enzymes, display decreased activities in the affected brain region in $\mathrm{AD}$ [19].

Diterpenes have been reported to be neuroprotective due to their ability to enhance antioxidant enzymes that lead to a decrease in the generation and accumulation of ROS. Forskolin is a naturally occurring diterpene widely distributed in various parts of plants. Forskolin have been reported to have diverse pharmacological properties such as antiinflammatory, antimutagenic and anticarcinogenic activity [21, 22]. However FL has not been evaluated for effects against scopolamine-induced cognitive impairment in rodents. Therefore, present study, assessed the effect of forskolin against scopolamineinduced amnesia in rats. Antioxidant activity is wellknown biological activity of Forskolin [23]. It contains some only one of its kind functional groups; important among them is tetrahydropyran derived heterocyclic ring [21]. Tetrahydropyran derived heterocyclic ring is synthesized after the generation of the trans-fused carbon ring are formed by a carbocation mediated cyclization [21]. This study, investigated the antioxidant effect of Forskolin against scopolamineinduced cognitive impairment in rats.

Scopolamine, a muscarinic antagonist which has ability to block central cholinergic receptors, causes a reversible impairment of both (i) maintaining attention; (ii) the processing of information as well as the acquisition of new knowledge in rodents and humans. The cognitive degradation seen resembles the memory disturbances seen in Alzheimer's disease. Hence, scopolamine-induced cognitive impairment has been repeatedly used as an experimental model for AD. It has been recently, reported that cognitive impairment in the scopolamine-induced rat model is associated with enhanced ROS production within the rat brain. Scopolamine affects memory and cognitive function, and subsequently causes harm to long-term and shortterm memories. It has been previously reported that cognitive destruction caused by acute scopolamine administration in rodents is marked by changed levels of GSH in the brain and altered activities of antioxidant enzymes. Scopolamine treatment resulted in a significantly elevated TBARS level, hallmark of lipid peroxidation, and significantly reduced enzymatic activity of glutathione reductase and acetylcholinesterase in the cortical and hippocampal region of amnestic rat [15].
Therefore, in the present study rat with scopolamine-induced amnesia was used as the model for investigating whether forskolin could effectively reverse scopolamine-induced memory deficits in rats. Scopolamine treatment affected performances on several memory tasks like total no. of entries and spontaneous alteration in Y-maze. Furthermore, scopolamine disrupted the recognition of objects in NORT, a test widely used to examine recognition performance in rats. These observations on behavioral models suggested that scopolamine had produced impairment in learning as well as memory. Memory impairment induced by scopolamine in rat is characterized by increased oxidative stress within central nervous system [15-17] which also has been indicated in earlier studies.

However, administration of Forskolin and Donepezil (Standard drug) successively for 14 days to rats significantly restored the memory as indicated by improvement in memory scores of these behavioral models. Scopolamine-induced memory impairment was successfully reversed by Forskolin $750 \mu \mathrm{g} / \mathrm{kg}$ $(* * * \mathrm{P}<0.001), \quad 500 \mu \mathrm{g} / \mathrm{kg} \quad(* * \mathrm{P}<0.01), \quad 250 \mu \mathrm{g} / \mathrm{kg}$ $\left({ }^{\mathrm{NS}} \mathrm{P}<0.05\right)$ as indicated by increased total no. of entries and spontaneous alteration in $\mathrm{Y}$-maze and increase in exploration towards novel object.

Pre-treatment with Donepezil (3 $\mathrm{mg} / \mathrm{kg}$ ) reversed scopolamine-induced memory deficits $(* * * \mathrm{P}<0.001)$ similar to previously reported studies.

Oxidative stress is involved in almost all pathophysiological cerebral changes. Oxidative stress causes the production of ROS. As the production of ROS increases in turn leads to rise in lipid peroxidation and reduced antioxidant enzymes leads to decreased scavenging of free radicals, which has been reported in Alzheimer's disease brain [24]. Scopolamine significantly raised oxidative stress level as indicated by the increased thiobarbituric acid reactive substances and increased acetylcholinesterase levels along with decreased reduced -glutathione.

Once daily administration of forskolin for two weeks decreased oxidative stress as well as it also prevented the scopolamine-induced rise in oxidative damage as indicated by the reduced thiobarbituric acid reactive substances, increased glutathione and acetylcholinesterase levels as compared to control group.

Effect on behavioral and biochemical parameters suggest potential of forskolin in the treatment of Alzeimer's disease. 


\section{CONCLUSION}

From results of this study, it is concluded that forskolin enhances cognitive performances in rats with scopolamine-amnesia.

These findings gives insight into development of phytochemicals for the treatment of conditions associated with a neurodegenerative disorder of Alzheimer's type.

\section{REFERENCES}

1. Anekonda, T. S., \& Reddy, P. H. (2005). Can herbs provide a new generation of drugs for treating Alzheimer's disease?. Brain research reviews, 50(2), 361-376.

2. Alzheimer's Association. (2013). 2013 Alzheimer's disease facts and figures. Alzheimer's \& dementia, 9(2), 208-245.

3. Laurence, L. B., Keith, L. P., Donald, K. B., \& Iain, L. O. B. (2008). Goodman and Gilman's manual of pharmacology and therapeutics.

4. Yoo, K. Y., \& Park, S. Y. (2012). Terpenoids as potential anti-Alzheimer's disease therapeutics. Molecules, 17(3), 3524-3538.

5. Hong, S., Kim, S. H., Rhee, M. H., Kim, A. R., Jung, J. H., Chun, T., ... \& Cho, J. Y. (2003). In vitro anti-inflammatory and pro-aggregative effects of a lipid compound, petrocortyne A, from marine sponges. Naunyn-Schmiedeberg's archives of pharmacology, 368(6), 448-456.

6. Mollica, A., Locatelli, M., Stefanucci, A., \& Pinnen, F. (2012). Synthesis and bioactivity of secondary metabolites from marine sponges containing dibrominated indolic systems. Molecules, 17(5), 6083-6099.

7. Sagar, S., Kaur, M., \& Minneman, K. P. (2010). Antiviral lead compounds from marine sponges. Marine drugs, 8(10), 2619-2638.

8. Lysek, N., Kinscherf, R., Claus, R., \& Lindel, T. (2003). L-5-Hydroxytryptophan: Antioxidant and Anti-Apoptotic Principle of the Intertidal Sponge Hymeniacidon heliophila. Zeitschrift für Naturforschung C, 58(7-8), 568-572.

9. Saleem, A. M., Dhasan, P. B., \& Rafiullah, M. R. M. (2006). Simple and rapid method for the isolation of forskolin from Coleus forskohlii by charcoal column chromatography. Journal of Chromatography A, 1101(1-2), 313-314.

10. Lukhoba, C. W., Simmonds, M. S., \& Paton, A. J. (2006). Plectranthus: a review of ethnobotanical uses. Journal of ethnopharmacology, 103(1), 1-24.

11. Alasbahi, R. H., \& Melzig, M. F. (2010). Plectranthus barbatus: a review of phytochemistry, ethnobotanical uses and pharmacology-part 1. Planta medica, 76(07), 653-661.

12. Seamon, K. B., Padgett, W., \& Daly, J. W. (1981). Forskolin: unique diterpene activator of adenylate cyclase in membranes and in intact cells. Proceedings of the National Academy of Sciences, 78(6), 3363-3367.

13. Ando, S., Kametani, H., Osada, H., Iwamoto, M., \& Kimura, N. (1987). Delayed memory dysfunction by transient hypoxia, and its prevention with forskolin. Brain research, 405(2), 371-374.

14. Terry Jr, A. V., Callahan, P. M., Hall, B., \& Webster, S. J. (2011). Alzheimer's disease and age-related memory decline (preclinical). Pharmacology Biochemistry and Behavior, 99(2), 190-210.

15. Biradar, S. M., Joshi, H., \& Chheda, T. K. (2012). Neuropharmacological effect of Mangiferin on brain cholinesterase and brain biogenic amines in the management of Alzheimer's disease. European journal of pharmacology, 683(1-3), 140-147.

16. Cai, M., Shin, B. Y., Kim, D. H., Kim, J. M., Park, S. J., Park, C. S., .. \& \& Ryu, J. H. (2011). Neuroprotective effects of a traditional herbal prescription on transient cerebral global ischemia in gerbils. Journal of ethnopharmacology, 138(3), 723-730.

17. Ennaceur, A., \& Delacour, J. (1988). A new onetrial test for neurobiological studies of memory in rats. 1: Behavioral data. Behavioural brain research, 31(1), 47-59.

18. Moron, M. S., Depierre, J. W., \& Mannervik, B. (1979). Levels of glutathione, glutathione reductase and glutathione $S$-transferase activities in rat lung and liver. Biochimica et Biophysica Acta (BBA)-General Subjects, 582(1), 67-78.

19. Andersen, J. K. (2004). Oxidative stress in neurodegeneration: cause or consequence?. Nature medicine, $10(7 \mathrm{~s}), \mathrm{S} 18-\mathrm{S} 25$.

20. Technical abcam lab ab138871 Acetylcholinesterase Assay Kit (Colorimetric) Abcam 2012.

21. Insel, P. A., \& Ostrom, R. S. (2003). Forskolin as a tool for examining adenylyl cyclase expression, regulation, and $\mathrm{G}$ protein signaling. Cellular and molecular neurobiology, 23(3), 305-314.

22. Wagh, V. D., Patil, P. N., Surana, S. J., \& Wagh, K. V. (2012). Forskolin: upcoming antiglaucoma molecule. Journal of postgraduate medicine, 58(3), 199-202.

23. Erdogan, S., Aslantas, O., Celik, S., \& Atik, E. (2008). The effects of increased cAMP content on inflammation, oxidative stress and PDE4 transcripts during Brucella melitensis infection. Research in veterinary science, 84(1), $18-25$.

24. Jomova, K., Vondrakova, D., Lawson, M., \& Valko, M. (2010). Metals, oxidative stress and neurodegenerative disorders. Molecular and cellular biochemistry, 345(1-2), 91-104. 